\title{
Identification of the benefits, enablers and barriers to integrating junior pharmacists into the ward team within one UK-based hospital
}

This article was published in the following Dove Press journal:

Integrated Pharmacy Research and Practice

13 November 2017

Number of times this article has been viewed

\author{
Man Yui Hung' \\ David John Wright ${ }^{2}$ \\ Jeanette Blacklock ${ }^{2}$ \\ Richard John Needle ${ }^{1,2}$
}

'Pharmacy Department, Colchester Hospital University NHS Foundation Trust, Colchester, ${ }^{2} \mathrm{School}$ of Pharmacy, University of East Anglia, Norwich, UK
Introduction: A high nurse-vacancy rate combined with high numbers of applications for junior pharmacist roles resulted in Colchester Hospital University National Health System Foundation Trust trial employing junior pharmacists into traditional nursing posts with the aim of integrating pharmacists into the ward team and enhancing local medicines optimization. The aim of the evaluation was to describe the implementation process and practice of the integrated care pharmacists (ICPs) in order to inform future innovations of a similar nature.

Methods: Four band 6 ward-based ICPs were employed on two wards funded within current ward staffing expenditure. With ethical committee approval, interviews were undertaken with the ICPs and focus groups with ward nurses, senior ward nurses and members of the medical team. Data were analyzed thematically to identify service benefits, barriers and enablers. Routine ward performance data were obtained from the two ICP wards and two wards selected as comparators. Appropriate statistical tests were performed to identify differences in performance.

Results: Four ICPs were interviewed, and focus groups were undertaken with three junior nurses, four senior nurses and three medical practitioners. Service enablers were continuous ward time, undertaking drug administration, positive feedback and use of effective communication methods. Barriers were planning, funding model, career development, and interprofessional working and social isolation. ICPs were believed to save nurse time and improve medicines safety. The proportion of patients receiving medicine reconciliation within 24 hours increased significantly in the ICP wards. All ICPs had resigned from their role within 12 months.

Discussion: It was believed that by locating pharmacists on the ward full time and allowing them to undertake medicines administration and medicines reconciliation, the nursing time would be saved and medicines safety improved. There was however significant learning to be derived from the implementation process, which may enable similar future models to be introduced more successfully.

Keywords: pharmacist, ward team, medicines administration, medicines reconciliation

\section{Introduction}

In 2014-2015, there were 18.7 million inpatient episodes recorded in all National Health System (NHS) hospitals in England, up from 18.2 million the year before with over $60 \%$ involving some form of medical procedures and interventions. ${ }^{1}$ While medicines remain the most common therapeutic intervention within Western health care through illness prevention, acute and chronic illness management and maintenance of health and quality of life, poor medicines management is associated with compromised patient safety, increased hospital admissions and prolonged length of hospital stay. ${ }^{2}$
Correspondence: David John Wright School of Pharmacy, University of East Anglia, Norwich Research Park, Norwich NR4 7TJ, UK

Tel +44I603592042

Email D.j.wright@uea.ac.uk 
Medication errors can happen at any stage of the medicines use process, starting from prescribing, preparing, dispensing, administering and monitoring, to providing relevant advice on medicines to patients. In 2010-2015, there were over 500,000 medication incidents reported to the National Reporting and Learning System in England and Wales, of which 75\% were from acute general hospitals. Of these reported incidents, $0.9 \%$ resulted in death or severe harm. ${ }^{3}$

It is recognized that pharmacists play an important role in identifying these potential errors and preventing harm from such incidents, of which the majority are "avoidable" ${ }^{4,5}$ Within the NHS, it is estimated that up to $£ 770$ million could be saved from the cost related to all medication errors. ${ }^{5}$ In recognition of the need to ensure that the maximal benefit is derived from medication, one of the current government agendas in the UK is medicines optimization, ${ }^{6}$ which is driving change within the role of pharmacists in both the hospital ${ }^{7,8}$ and community settings. ${ }^{9}$ Pharmacy input to the ward is supported and welcomed by other multidisciplinary health care professionals including nurses and doctors, due to pharmacists' extensive knowledge in pharmacology and the associated contributions to medication safety. ${ }^{10}$ The limited availability of pharmacy services outside core hours is often associated with an increase in missed doses and prescription errors, possibly due to the lack of medicines reconciliation or it being done poorly. ${ }^{11,12}$ In addition, it is also associated with a higher incidence of delayed discharge and unnecessary length of stay in hospital due to waiting for discharge medication. ${ }^{11,12}$

In England, it is usual practice for hospital wards to receive regular pharmacist visits to undertake a variety of clinical activities including medicines reconciliation, review of medication charts, reducing omitted doses, reporting of medicines-related incidents, patient counseling, pre- and post-admission clinical assessment and antimicrobial stewardship. ${ }^{13}$ While the time allocated to this activity varies widely between hospital trusts, it is unusual for hospital pharmacists to be located entirely on the ward or line managed within the ward team. Consequently, hospital pharmacists in England are not routinely integrated into ward teams in the same manner as doctors and nurses whose responsibilities and line management are primarily located within the ward. A recent review of hospital services in England recommended that at least $80 \%$ of hospital pharmacist time should be spent undertaking patient-facing activities as this was believed to provide greater value to the NHS than pharmacist involvement in medicines supply activities where they were identified as currently spending almost half of their time. ${ }^{13}$ While the term "patient facing" has not been defined by the government, it suggests traditional clinical pharmacist activities, which are undertaken in front of patients. Consequently, new models of hospital pharmacy service delivery are required in the UK.

The inability to recruit nurses into traditional wardbased nurse roles resulted in Colchester Hospital University National Health System Foundation Trust (CUHFT) deciding to replace some of the posts with relatively new qualified pharmacists, where application numbers were relatively high. The integrated care pharmacist (ICP) posts were created to allow pharmacists to deliver extended hours service on the wards and to assume more local ownership of the medicines optimization agenda (medicines administration, medication review, medicines reconciliation, discharge planning) while undertaking a number of traditional nursing activities (intravenous cannulation, injection preparation and some basic physical health observations, for example, blood pressure, pulse monitoring and oral care). It was believed that by undertaking these elements of the nursing role, it would enable nursing staff to allocate their time to other activities. ${ }^{14}$ The innovative service model was seen as one possible approach to meeting the government proposals for pharmacists to spend the majority of their time in patient-facing roles. ${ }^{13}$

Four junior-grade pharmacists were employed on two pilot wards on a shift pattern basis, 7 days a week (excluding night shifts). The roles were managed by the ward sister, as part of the ward establishment and funded and created within the current ward staffing expenditure. The roles were initially fixed as 2-year internships for newly qualified pharmacists, underpinned with a competency program and professionally mentored by a senior nurse and a senior pharmacist. The roles did not replace the current ward pharmacist role of visiting the ward daily, but instead were intended to be an integral part of the ward template. ICPs took up their roles at CUHFT in September 2015 on an elderly care and respiratory ward. The aim of the evaluation was to describe how the model was implemented and the activities undertaken by the ICPs and identify any perceived or actual changes in ward performance.

\section{Methods}

This research project was funded by Health Education England and sponsored by the University of East Anglia (UEA). Approval for the research was received from the UEA Faculty of Medicine and Health Sciences Ethics Committee. Local approval was obtained for the study to be conducted on CUHFT premises.

\section{Qualitative research}

Qualitative data collection took place 10 months after the new role was established. 
Focus groups were selected as the method for data collection as this approach allows participants to provide in-depth data on their experience with the ICPs, enables consensus and dissonance to be identified and as a group activity stimulates discussion. ${ }^{15}$

All participants were selected from the two inpatient wards where the ICPs were located. Staff nurses, that is, those who are not in management roles, senior nurses in management roles and doctors were all approached for consent to participate separately in their own focus group. All participants provided written informed consent to participate in this study. Due to ICP resignations from the role, individual interviews were undertaken instead of the planned focus groups.

The day before the focus groups/interviews, all participants were contacted via telephone to remind them of the venue details in order to optimize attendance. Refreshments were provided to encourage attendance. The ground rules of participating in a focus group were explained to the participants, including the purpose of recording, note taking and data protection. All interviews (including telephone interviews) and focus groups were taken place in a private location at Colchester General Hospital at a time deemed to be most convenient for the professionals who attended. The focus groups were chaired and moderated by two researchers. All group meetings and interviews were recorded using a digital recorder and transcribed verbatim by Man Yui Hung (MYH).

The topic guide stem questions for both interviews and focus groups were as follows:

- Describe the activities undertaken by the ICPs

- Identify the barriers and facilitators to the role

- Describe views on the role

- Identify any differences in ward performance as a result of the new role

Data from focus groups and interviews were analyzed using a thematic analysis. ${ }^{16}$ The transcripts were read repetitiously, and initial ideas were noted down. Second, interesting features of the data were manually coded in a systematic manner, and all data extracts were collated together within each code. The third phase involved sorting these codes into potential themes and gathering all data relevant to each potential theme. Subsequently, these themes were reviewed and refined. The entire data set was re-read to ensure the validity of the potential themes and to capture any additional data within themes, which might have been missed in preceding coding stages. The subsequent fifth phase entailed ongoing analysis to refine the specifics of each theme. MYH then reviewed the collated data extracts for each theme, organized them into a coherent and consistent account and generated clear definitions and names for each theme. The last step of the process provided the final opportunity for analysis, which involved producing a report including a selection of compelling extract examples and an analytic narrative.

\section{Quantitative research}

Routinely collected process data were collated from the two wards involved in the new service, and comparative data collected from two wards of a similar profile in terms of size, age of patients and type of ward. Data were collected from September 2014 until May 2015 and then from September 2015 until May 2016 (when the ICPs started their service). Only 9-month data were collected due to ICP resignations which occurred after the first 6 months, and by 9 months, only two ICPs remained in post.

The following process data were collected for the 9 months of the ICP service and the same 9 months the year before:

- medicines reconciliation (ensuring that medicines-related information regarding the patient in secondary care aligns with the information in primary care) performed on ward within 24 hours of patient admission

- length of stay and readmission rates

- drug spend per ward

Independent samples $t$-test and Mann-Whitney $U$-test (dependent on data distribution) were used to perform comparisons between ICP and comparator wards. Paired samples $t$-tests and Wilcoxon matched pairs test were used to compare before and after data in each setting. Fisher's exact was used to compare proportions.

\section{Results}

Individual interviews were conducted with the two ICPs in post, and telephone interviews conducted with the two ICPs who had left the Trust. Focus groups were carried out with the following three groups of professionals:

- $\quad$ staff nurses - three participants

- senior nurses in management roles - four participants

- medical team including registrars and consultants three participants

\section{Themes}

Themes were identified and organized into barriers, enablers and perceived benefits. The enablers were continuous ward 
time, involvement in drug administration, positive feedback and support and utilization of effective communication methods. The barriers were planning and implementation, financial arrangements, career development and trajectory, and interprofessional working and social isolation. Perceived benefits were nurse time, ward staff support and education and medicines optimization.

\section{Enablers}

\section{Continuous ward time}

Increased time on ward, which allowed the delivery of a better clinical service, was seen as a positive element to the role:

You can actually monitor the patient and see if it has any clinical effect on the patient, so hmm you can follow through a patient very easily cos you are there three days a week. [ICP]

\section{Involvement in drug administration}

Being involved in drug administration was seen as good for personal development and future support purposes:

It's good that we do drug administration, because especially on our diploma days [...] you're learning about PEG feeds, what type of NG tubes and what sizes and stuff, if you haven't seen it in real life [...] what's the point of learning it [...] I do think the drug administration has been beneficial cos it's been an eye opener.... [ICP]

\section{Positive feedback and support}

Positive feedback and support from colleagues/patients/relatives was identified as facilitating role delivery. The use of a communication book was seen as a method of improving role effectiveness.

\section{Utilization of effective communication methods}

We had communication books so if we had things we needed them, you know, we'd write them down in a book [...] different bits like discharge tomorrow so that they'd know as well $[\ldots]$ and then they'd just tick off when they'd done all the bits.... [Nurse]

\section{Barriers}

\section{Planning and implementation}

Problems with initial planning and implementation were seen as creating barriers from the outset.

ICPs and other health care professionals repeatedly reported that the job description, which was a combination of nurse and pharmacist roles, was too great.
I think it was quite a difficult role [...] I think that we [...] the job role was too big for the one individual person and we'd had several meetings about this cos they could not fulfil the whole role that they were expected to do.... [Senior nurse]

The outcome from this was that the ICPs felt overworked with too many tasks:

[...] there was a lot for them to do. And when we are short staffed we are asking them to do something [...] so many things and they can't help us with everything or they can't do everything that they have to do.... [Staff nurse]

The lack of clear definition and management of expectation resulted in different expectations of the role from the ICPs and different ward staff:

they should not have any sort of misconceptions of what their role is, so everything should be in black and white and all team members should know exactly what is the role of the (ICP) and what's the extent of the role of these interns. I think that was the main major barrier that they didn't seem to know and we didn't seem to know where to draw the line. [Doctor]

Careful consideration of the job description for ICPs was recommended. Involvement of ward staff in the setting up and delivery of the service was seen as too late, and this may have contributed to the confusion and misconceptions from the outset:

It would have been nice if we were involved a bit earlier [...] I wasn't even [...] I didn't know this was happening until the people were appointed. It felt as though we didn't get the support $[\ldots]$ so it needed to be $[\ldots]$ you needed to have support there. [Senior nurse]

Promotion of the role to all staff from the outset was seen as helping with integration.

\section{Financial arrangements}

Funding arrangements were seen as a significant barrier with the fact that the ICPs were employed with nursing funding causing disquiet when they were not undertaking many of the nursing roles they have replaced:

I think the funding was from the nursing staff wasn't it $[\ldots]$ ? So I think there was a feeling that you know they're (the ward) not really getting value for money [...] for what they (the ward) are paying basically [...] cos they (ICPs) were doing only the pharmacy bit in a sense so there were other things which the nurses do like patient care and a lot of time [...]. [Doctor] 
There was a consensus that the role should not be funded from the nursing budget. The fact that the ICPs were employed at a higher band than those whom they were replacing created greater expectations which were not met:

I appreciate that they're coming in as a pharmacist so they're coming in as a six (Reference to level of pay) so I think that some of the staff felt that why are they [...] as awful as it sounds, why are they being $x$ amount when they're not actually doing half of what they're supposed to be doing. So I think there was a little bit of that, not really so much now $[. .$.$] maybe initially to start with [. .$.$] a lot of people$ were saying why has this not been done [...]. [Senior nurse]

\section{Career development and trajectory}

The planned education and training was identified as a barrier to the development and integration of the ICPs. This was particularly pertinent to the induction process.

The induction was way too long and complicated. I mean I think it was over two months until they actually started properly and it was way too much to what they needed. [Senior nurse]

The selection of pharmacists for role who already had experience of hospital practice was identified as one approach to addressing some of the education needs.

It would be better if perhaps they had some hospital experience. [Senior nurse]

They should know how the ward functions [...] what sort of roles of the key members and then they should be able to establish what exactly they can take over [...]. [Doctor]

A lack of shared responsibility between pharmacy and ward to manage and mentor ICPs was identified as a barrier.

I still believe that this is a brilliant idea and I wish it was probably thought through a bit more or approached a little bit better and I think it's really important that if the responsibility for the role is split between pharmacy and the ward $[\ldots]$ because of the dynamics and the understanding of the role, it's not fair on the ward team to accept what a pharmacist does but also to leave that person on their own without support from pharmacy team. So if anybody ever decides to start this (programme) again, this would have to be a shared responsibility (to manage the ICPs). [ICP]

There was a feeling that the role had no clear career trajectory and that there was no fulfillment from undertaking the same roles every day:
At the moment if anything [...] I think I have regressed than [...] in most of my skill cos it just seems as though most of my time and skills (have been) spent on discharges rather than actually looking through a patient's notes and actually looking at a drug chart $[\ldots]$. [ICP]

\section{Interprofessional working and social isolation}

The ICPs believed that there may have been some interprofessional barriers in place before the pharmacists started within their roles:

I think barriers [...] [inaudible] [...] I think the biggest one is, more in a social aspect hmmm [...] even from the start it was very [...] [inaudible] I think it seems like most [...] like the band 5 nurses weren't appreciative of having a like, pharmacist on the ward. [ICP]

One of the ICPs reported feeling isolated from both the pharmacy team and the ward team and that this requires consideration when setting up such posts:

Obviously I do have people who come and help me on the ward regularly which is great because there's plenty to do but at the same time I feel quite isolated from the pharmacy team but I'm not really part of the ward team either [...] I mean I am (part of the ward team) as a person but as a role I don't think it's integrated as much as it probably should have been if that makes sense. [ICP]

\section{Benefits}

There was recognition that the role provided some value, and suggestions for how it could be continued were made.

\section{Nurse time}

There was recognition that the role did relieve some of the time pressures experienced by nurses:

One thing I would say it the difference I've noticed since the ICPs have left is that now when we want drugs at short notice we struggle to get them [...] we have to try and put things in place, whereas the ICPs would themselves out and actually run down to pharmacy to go and get them because they would be on the ward so if we had so. [Senior nurse]

\section{Ward staff support and education}

It was believed that the ICPs improved medication safety:

The discharges were [...] I reckon safer because all the drugs were ordered, everything was up, you know everything was ready, the clinical checks were done, they would help with the clinical checks [...]. [Senior nurse] 
The ICPs were also seen as improving ward education regarding medicines:

[...] I feel safer when I ask them because they are from pharmacy and they know better than me. I feel that it's more safety for patients because then I know for sure about the medication [...] and if they (ICP) don't know, they know where to look up the information and they are quicker than me. [Staff nurse]

\section{Medicines optimization}

The ICPs believed that they were effective at reducing waste:

Like stock in the cupboard [...] I started marking with open boxes so that the nurses actually hmmm [...] started using the open box rather than. [ICP]

The medicines-related support "out of hours" was appreciated:

As I said [...] again very useful having them out of hours as well cos often decisions, unfortunately, can be made late in the day and then you can't get hold of a pharmacist which means obviously the junior doctor has to authorise the meds (medicines) and say [...] so from that aspect it's probably safer that someone else had checked the discharge summary before that patient goes home even though the decision was made very late that they could go home. [Doctor]

\section{Quantitative research}

Medicines reconciliation

Figure 1 shows the level of medicines reconciliation performed pre- and post-ICP employment. It can be seen that while the level of medicines reconciliation remained relatively static within the two comparison wards, it increased by $>6 \%$ in both of the ICP wards, with this difference being significant in ward 2. When the two ICP wards are combined, the overall difference is still significant ( $p<0.001$, Fisher's exact test).

\section{Readmission rates}

Table 1 shows readmission rates for the ICP and comparison wards. It can be seen that no differences were identified.

Table 2 shows that while both ICP wards showed reduction in the length of stay and the variation in the length of stay, this was not significant. Within the comparison wards, there was a very small increase in one ward and a significant reduction in the other of 4 days. Reasons for this were not elucidated.

\section{Discussion}

The project evaluation was performed to enable others to learn from the experience and to inform any future evaluation to ensure that the most appropriate data would be captured. The qualitative results identified a number of perceived

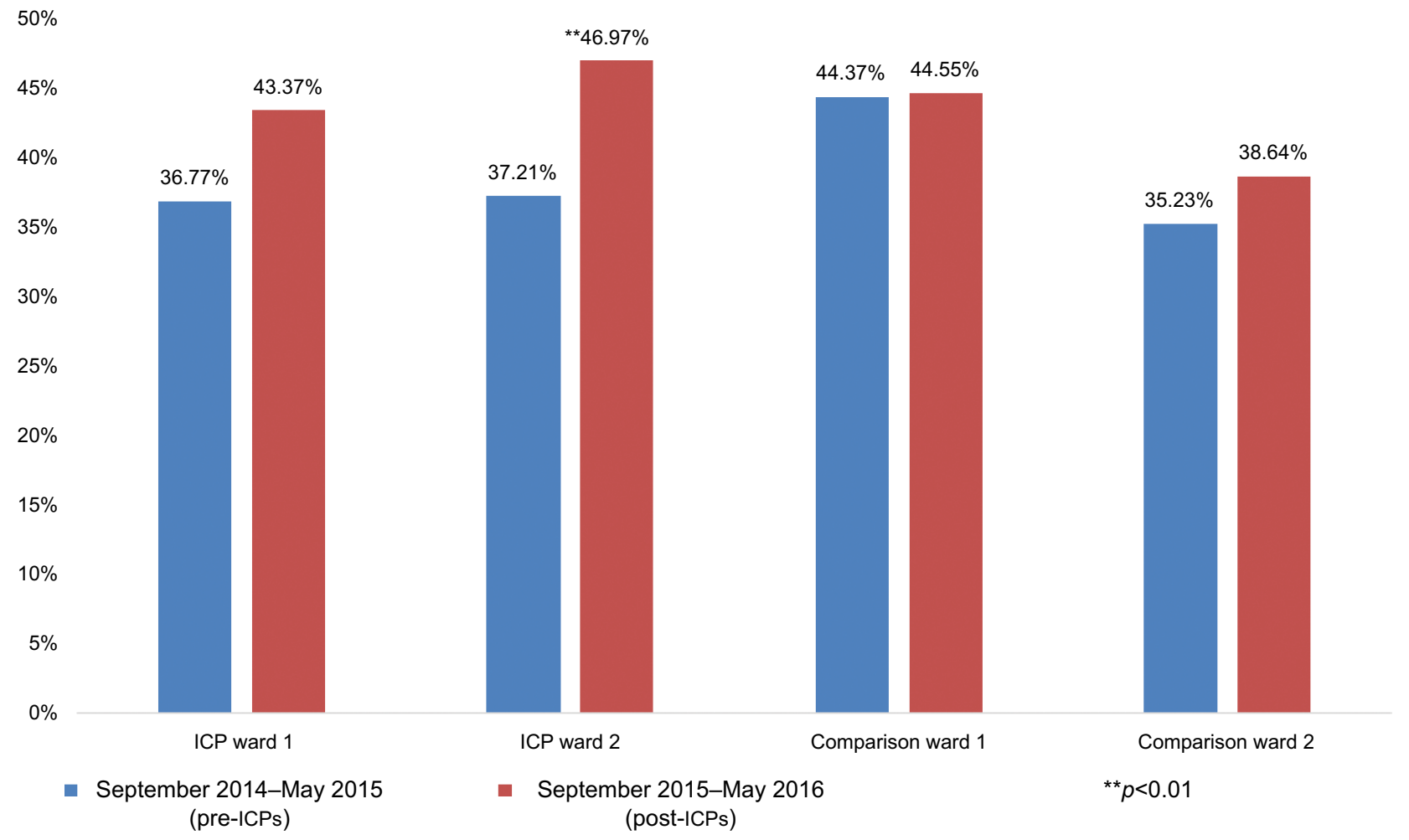

Figure I Medicines reconciliation within 24 hours of admission time. Abbreviation: ICP, integrated care pharmacist. 
Table I Comparison of readmission rates (within 30 days) between ICP and comparison wards

\begin{tabular}{llll}
\hline Wards & Mean (SD) \% readmission rate & Paired samples t-test \\
\cline { 2 - 4 } \cline { 2 - 4 } & Pre-ICPs (September 20 I4-May 20 I5) & Post-ICPs (September 20 I 5-May 20 I6) & P-value \\
\hline Comparison wards & I7.I (5.8) & $19.3(6.4)$ & 0.375 \\
Intervention wards & I5.9 (4.2) & $17.4(4.8)$ & 0.192 \\
ICP ward I & I4.8(4.5) & $17.4(5.7)$ & 0.24 \\
Comparison ward I & I6.I (7.2) & $20.1(7.1)$ & 0.354 \\
ICP ward 2 & I6.9 (3.7) & $17.4(4.1)$ & 0.634 \\
Comparison ward 2 & I8.I (4.I) & $18.4(5.9)$ & 0.892 \\
\hline
\end{tabular}

Abbreviation: ICP, integrated care pharmacist.

Table 2 Comparison of mean length of stay between ICP and comparison wards

\begin{tabular}{|c|c|c|c|}
\hline \multirow[t]{2}{*}{ Wards } & \multicolumn{2}{|l|}{ Mean (SD) length of stay in days } & \multirow{2}{*}{$\begin{array}{l}\text { Paired samples } t \text {-test } \\
p \text {-value }\end{array}$} \\
\hline & Pre-ICPs (September 20 I4-May 20I5) & Post-ICPs (September 20 I5-May 2016) & \\
\hline Comparison wards & II.7 (4.4) & $9.9(1.6)$ & 0.052 \\
\hline Intervention wards & $12.6(2.3)$ & $11.7(2.1)$ & 0.145 \\
\hline ICP ward I & $14(1.6)$ & I3.4 (0.7) & 0.37 \\
\hline Comparison ward I & $8.5(2.5)$ & $8.8(1.0)$ & 0.761 \\
\hline ICP ward 2 & II.I (2) & $10.1(1.6)$ & 0.284 \\
\hline Comparison ward 2 & $14.8(3.6)$ & $10.9(1.5)$ & 0.006 \\
\hline
\end{tabular}

Abbreviation: ICP, integrated care pharmacist.

benefits from integrating pharmacists into ward teams such as improved medicines safety through increased medicines reconciliation, more immediate support for ward staff from being available outside of usual hours and improving medicinesrelated culture through staff support and education.

While this was an innovative idea, which seemed a sensible solution to the identified problem at the time, the resignation of all of the ICPs before the end of their contracts suggests that the role as set up and implemented was unattractive to the ICPs. The fundamental problems seem to have been the use of nursing funding to underpin the role, unrealistic and possibly unfair expectations due to the nature of the job description, lack of ward staff understanding of the role in advance and tensions due to the different pay bandings. Consequently, significant changes would be required for it to have any chance of sustainable success in the future.

Implementation of the role could have been improved with a more clearly defined, disseminated job description and an integrated induction process, as this would have allowed the ICPs to become part of the ward team much earlier. The tasks, which the ICPs ultimately focused on, were the admission and discharge processes, and, due to the limited nature, were not seen as the basis for an attractive long-term role. Involvement in the medication administration process was seen as valuable to both ward staff and the ICPs as was their impact on the medicines culture within the wards, and therefore, these are elements of this role and process which may be useful for repetition.
The only measurable outcome that demonstrated a meaningful difference as a result of the introduction of ICPs was the proportion of medicines reconciliations completed within 24 hours. The relatively small increase seen in itself would not justify the amount of time spent by the ICPs on the ward, and from the ICP perspective, their time was largely spent on organizing discharges in order to reduce the average length of stay and enable beds to be filled more quickly. The small reduction in length of stay seen on both wards compared to the same time period the year before may be partly attributable to this; however, there are many factors associated with length of stay, and there is a general trend downward with respect to time spent in hospital by inpatients.

The use of a pharmacist who was independent of the project to undertake the evaluation from a qualitative perspective resulted in very honest and open responses, and as such, the derived data are likely to represent a high level of validity. Furthermore, the use of quantitative data, which are routinely collated, should minimize the chances of ward teams changing their natural behavior, and therefore, the data are unlikely to be biased due to observation effects.

The results for this study should however be viewed with some caution as it is based on the experience of employing four pharmacists on two wards only. Due to the effect of individual personalities and the complex nature of individual ward environments, it is unlikely that exactly the same results would be seen if the project was to be repeated. The number of individuals within the focus groups was small, and therefore, 
a full picture may not have been obtained from those individuals who consented. Furthermore, a before-and-after design with comparator wards which were not ideal comparators limits the usefulness of the quantitative data. It is not possible to delineate the effect of ICPs from trends, which are independently occurring, or from natural variation, which occurs within such environments.

The wide-ranging job description created different expectations from different stakeholders and uncertainty within the ICPs with respect to how to allocate their time. This was compounded by their lack of experience within the ward environment and in some cases hospital environment. Without bespoke training beyond induction, the ICPs operated in those areas, which are seen as traditional pharmacist roles. This created problems with the nurses on the ward who were expecting them to undertake nursing roles and hence relieve some of the workload problems associated with current nursing establishment. The problem was compounded by a lack of awareness of what the ICPs were expected to do and when they were meant to actually be present.

The thorough and extended induction, which had been planned for the ICPs, was not received positively due to the resulting delay in joining the ward team. It seems that inductions for new staff may be better staggered to enable them to be introduced to their role and the ward team at an earlier stage. Furthermore, the ward and pharmacy managers may want to consider how best to integrate such individuals into the ward team with the ICPs describing a sense of isolation within the role. Pharmacists tend to move around the country for new posts and career development, which is less commonly seen within nursing staff. Consequently, the ICPs joined wards where most staff will already have extensive local networks of colleagues and friends while the ICPs may have neither.

The evaluation suggests that the job description associated with the role required more consideration from the outset. Essentially as an amalgam of a junior pharmacist post and ward-based nurse role, it allowed the wards free range as to what they could expect from the individuals once in post. Unfortunately, the ICPs interpreted this job description as everything they should do, and different individuals around them focused on the elements they wanted/expected them to do. This difference in expectations resulted in dissatisfaction from all concerned. Greater communication of the job description and senior manager expectations regarding the role to the ward team from the outset may have helped to manage expectations. However, it is perhaps of greater importance to develop a more defined and appropriate job description as a clear description of pharmacist roles and expectations has been identified as central to facilitating teamwork. ${ }^{17}$

Greater involvement of ward staff in the creation of the job description and development of the training program may have helped with the identified communication problems and integration of the ICPs into the team. If the ward team had assumed ownership of the role and development of the ICPs, then the hostility seen toward them may have been diminished.

The fundamental problem underpinning the project was however the use of nursing establishment funding to create the posts. This unsurprisingly resulted in nursing staff expecting the ICPs to spend a greater proportion of their time on nursing activities. The ICPs, who were trained as pharmacists, would not be expected to have the same skill set as a nurse or the same focus while on the ward.

Interprofessional barriers unsurprisingly formed once the nurses realized that the new posts were not going to make the same contribution to the nursing workload as if they had appointed a traditional nurse. This was further compounded by the use of a higher pay band for the pharmacists, who were additionally all still in training. Pharmacists are traditionally paid higher salaries within the NHS due to their master's qualification. While this does not create ill feeling when they are undertaking pharmacist activities, it will do if they are expected to undertake a nursing role with nurse funding. With these seemingly unsurmountable obstacles, it is perhaps not appropriate for pharmacists to be directly funded from nursing establishment funding. If the roles of ICPs are seen as necessary, then they should be funded from a different budget and encouraged to focus on those activities.

Medicines reconciliation activities at both admission and discharge were repeatedly highlighted as the main activity that the ICPs undertook, and this was confirmed by the increase in the proportion of patients seen within 24 hours by a pharmacist on the ICP wards. Even with pharmacists covering the ward for 7 days a week and for extended hours, the proportion of patients seen within 24 hours did not reach anywhere near $100 \%$. The main explanation for this is that many patients are not admitted directly to the ICP wards, and therefore, the 24-hour window is frequently missed on another ward.

As a before-and-after-service evaluation without a truly comparable control, it is not unsurprising that no difference in either of these outcomes were seen during the study. Length of stay is reducing in all areas, and at a national level, it reduces the ability to detect any change as a result of the service within this evaluation. Similarly, as only a proportion of patients are 
receiving the service from the ICPs, it is unlikely that the effect is seen within ward-level data and therefore is unlikely to demonstrate a reduction in unplanned rehospitalization. The ICPs were however unsupportive of their main role being medicines reconciliation as they described this as deskilling them in the long term and not providing an ideal career trajectory.

The one activity which all stakeholders were positive about was involvement in medicines administration. This was seen as useful for training purposes for the ICPs and as an activity that contributed significantly to nursing workload. While pharmacists are seen as the experts in medicines, there is little focus on the physical administration of medicines within their training. By expecting pharmacists to administer medicines, they become aware of the difficulties experienced by nurses if the treatment and formulation choices are not optimized. Medicines administration is a relatively simple task, which, the results suggest, should be undertaken by pharmacists as part of their training. Not only will it develop their drug-related knowledge but will also introduce significant patient contact and interaction into their curricula.

The amount of time spent on the ward by the ICPs, in comparison with the traditional ward pharmacist role, was seen as beneficial as it enabled the ICPs to monitor patients and provide continuity of care. The time on the ward should enable the development of strong interprofessional working relationships; however, there were a number of reasons identified and discussed previously which explain why this did not occur.

A number of benefits were perceived to be associated with having a pharmacist on the ward as an integral member with respect to medicines safety and staff education regarding medicines, and these have been identified in previously reported similar international projects. ${ }^{17,18}$

\section{Conclusion}

The novel service innovation was designed to address nursing shortages and improve local medicines optimization. It was believed to improve patient safety through improved medicines reconciliation and provision of medicines-related education and support to ward staff. The main barriers to the role were the funding model, dissonance with respect to role expectations, professional isolation and inability to identify how the career would progress beyond this. While the role in itself was supported by senior managers, greater involvement from the pharmacy team in mentoring the ICPs was seen as necessary as well as better planning from the outset with respect to job description and induction process.

The ICP role provides an ideal training model for pharmacists as it enables them to develop not only their clinical skills but also their consultation skills and interprofessional working. ${ }^{18}$ Development of a preregistration training model, which is based upon the ICP role, is therefore worthy of consideration, as it would only require additional supervision from a senior pharmacist and could be seen to be making a worthwhile contribution to the ward team.

\section{Acknowledgments}

The research was funded through an education grant provided by Health Education England. The project was sponsored by the University of East Anglia.

\section{Author contributions}

MYH undertook the original literature review, protocol design, qualitative data analysis and synthesis. MYH reviewed and commented on all versions of the final paper. DJW was responsible for managing the project and supervising both MYH and JB. He was responsible for preparation of the first draft of the paper and subsequent revisions following feedback. JB was the research associate employed on the project from the outset and was responsible for the collation, analysis and presentation of the quantitative data. She reviewed and commented on all drafts of the paper. RJN was part of the team who developed and implemented the innovation within Colchester Hospital. He secured the project funding, provided support for all elements of the data collection and commented on drafts of the paper. All authors contributed toward data analysis, drafting and critically revising the paper and agree to be accountable for all aspects of the work.

\section{Disclosure}

The authors have no conflicts of interest in this work.

\section{References}

1. Health and Social Care Information Centre. Hospital episode statistics. Admitted patient care, England - 2014-15. Available from: http://content.digital.nhs.uk/catalogue/PUB19124/hosp-epis-stat-admi-summrep-2014-15-rep.pdf. Accessed January 15, 2016.

2. Duerden M, Avery T, Payne R. Polypharmacy and medicines optimisation: making it safe and sound. The King's Fund. Available from: https://www.kingsfund.org.uk/sites/files/kf/field/field_publication_file/ polypharmacy-and-medicines-optimisation-kingsfund-nov13.pdf. Accessed January 11, 2016.

3. Cousins DH, Gerrett D, Warner B. A review of medication incidents reported to the National Reporting and Learning System in England and Wales over 6 years (2005-2010). Br J Clin Pharmacol. 2012;74(4):597-604.

4. Medicines and Healthcare Products Regulatory Agency. Patient Safety Alert. Stage Three: Directive. Improving medication error incident reporting and learning. 2014. Available from: https://www.england.nhs. uk/wp-content/uploads/2014/03/psa-sup-info-med-error.pdf. Accessed January 15, 2016. 
5. Frontier Economics Ltd. Exploring the costs of unsafe care in the NHS: a report prepared for the Department of Health. 2014. Available from: http://www.frontier-economics.com/documents/2014/10/exploring-thecosts-of-unsafe-care-in-the-nhs-frontier-report-2-2-2-2.pdf. Accessed January 15, 2016.

6. Royal Pharmaceutical Society. Medicines optimisation: helping patients to make the most of medicines. Good practice guidance for healthcare professionals in England. 2013. Available from: https://www.rpharms.com/Portals/0/RPS\%20document\%20library/ Open\%20access/News/patients---making-the-most-of-medicines. pdf?ver=2017-04-04-105412-883. Accessed January 15, 2016.

7. NHS England. Commissioning for quality and innovation (CQUIN): 2014/15 guidance. 2014. Available from: https://www.england.nhs.uk/ statistics/wp-content/uploads/sites/2/2013/09/CQUIN-Guidance-201415-PDF-751KB.pdf. Accessed January 23, 2016.

8. Specialist Pharmacy Service. Collation of medicines optimisation CQUINS and quality indicators. 2013. Available from: https://www. sps.nhs.uk/articles/collation-of-medicines-optimisation-cquins-andquality-indicators/. Accessed January 22, 2016.

9. National Institute for Health and Care Excellence. Medicines optimisation: the safe and effective use of medicines to enable the best possible outcomes. 2015. Available from: https://www.nice.org.uk/guidance/ ng5/. Accessed January 15, 2016.

10. Adhikari R, Tocher J, Smith P, Corcoran J, MacArthur J. A multidisciplinary approach to medication safety and the implication for nursing education and practice. Nurse Educ Today. 2014;34(2):185-190.

11. NHS Improvement. Equality for all - delivering safe care seven days a week - case studies. 2012. Available from: http://webarchive.nationalarchives.gov.uk/20151103090356/http://www.nhsiq.nhs.uk/resource-search/ publications/nhs-imp-seven-days.aspx. Accessed February 22, 2016.
12. Royal Pharmaceutical Society. Seven day services in hospital pharmacy - giving patients the care they deserve. 2014. Available from: https://www.rpharms.com/resources/reports/seven-day-services-inhospital-pharmacy. Accessed February 19, 2016.

13. Lord Carter of Coles. Operational productivity and performance in English NHS Acute Hospitals: unwarranted variations. An independent report for the Department of Health by Lord Carter of Coles. 2016. Available from: https://www.gov.uk/government/publications/ productivity-in-nhs-hospitals. Accessed October 16, 2017.

14. National Institute for Health and Care Excellence. Safe staffing for nursing in adult inpatient wards in acute hospitals. 2014. Available from: https://www.nice.org.uk/guidance/sg1/chapter/1-recommendations \#setting-the-ward-nursing-staff-establishment. Accessed February 21, 2016.

15. Finch H, Lewis J, Turley C. Focus groups. In: Ritchie J, Lewis J, McNaughton-Nicholls C, Ormston R, editors. Qualitative Research Practice: A Guide for Social Science Students and Researchers. 1st ed. Thousand Oaks, CA: Sage Publications Ltd.; 2003:211.

16. Braun V, Clarke V. Using thematic analysis in psychology. Qual Res Psychol. 2006;3(2):77-101.

17. Makowsky MJ, Schindel TJ, Rosenthal M, Campbell K, Tsuyuki RT, Madill HM. Collaboration between pharmacists, physicians and nurse practitioners: a qualitative investigation of working relationships in the inpatient medical setting. J Interprof Care. 2009;23(2):169-184.

18. Noble C, Brazil V, Teasdale T, Forbes M, Billett S. Developing junior doctors' prescribing practices through collaborative practice: sustaining and transforming the practice of communities. $J$ Interprof Care. 2017;31(2):263-272.
Integrated Pharmacy Research and Practice

\section{Publish your work in this journal}

Integrated Pharmacy Research and Practice is an international, peer-reviewed, open access, online journal, publishing original research, reports, reviews and commentaries on all areas of academic and professional pharmacy practice. This journal aims to represent the academic output of pharmacists and pharmacy practice with particular focus on integrated care. All papers are carefully

\section{Dovepress}

peer reviewed to ensure the highest standards as well as ensuring that we are informing and stimulating pharmaceutical professionals. The manuscript management system is completely online and includes a very quick and fair peer-review system, which is all easy to use. Visit http://www.dovepress.com/ testimonials.php to read real quotes from published authors. 